\title{
Administration and Toxicity Profile of the Capizzi Interim Maintenance-Retrospective Study from a Tertiary Care Cancer Centre
}

\author{
Aarthi Viswanathan ${ }^{1} \quad$ Arun Kumar $^{1} \quad$ Prakruthi S. Kaushik $^{1} \quad$ Avinash Thumallapalli $^{1} \quad$ C. Ramachandra $^{2}$ \\ B. S. Aruna Kumari ${ }^{1}$ Lingappa Appaji ${ }^{1}$ Nuthan Kumar ${ }^{1}{ }^{10}$
}

${ }^{1}$ Department of Pediatric Oncology, Kidwai Memorial Institute of
Oncology, D.H. Marigowda Road, Bengaluru, Karnataka, India
2 Department of Surgical Oncology, Kidwai Memorial Institute of
Oncology, D.H. Marigowda Road, Bengaluru, Karnataka, India

Ind J Med Paediatr Oncol 2021;42:426-430.

\begin{abstract}
Address for correspondence Nuthan Kumar, Department of Pediatric Oncology, Kidwai Memorial Institute of Oncology, D.H. Marigowda Road, Bengaluru, 560029, Karnataka, India (e-mail: nuthanraga28@gmail.com).
\end{abstract}

\begin{abstract}
Introduction The Capizzi-style methotrexate (MTX) is an integral part of acute lymphoblastic leukemia (ALL) treatment. The escalating dose of MTX originally used in the United Kingdom and Children's Oncology Group protocols along with Lasparaginase has been modified in the Indian Childhood Collaborative Leukemia (ICiCLe) group protocol where L-asparaginase has been omitted. The data regarding the incidence of toxicities and ease of administration on the Capizzi-style interim maintenance is not robust.

Objectives We have compiled our experience with administration and toxicity profile in children with intermediate-risk ALL.

Materials and Methods A retrospective data collection of all children diagnosed with intermediate-risk ALL as per the ICCCLe risk stratification in the year 2019 was included in the analysis. Each cycle of MTX was started after ensuring an absolute neutrophil count of $>750 / \mathrm{mm}^{3}$ and transaminases $<2$ upper limit of normal. As a unit protocol, pre- and postMTX hydration was administered in all our children. No urine $\mathrm{pH}$ or midcycle biochemical parameter monitoring was done. Statistical analysis was done using Microsoft Excel and SPSS version 24 IBM Corp. in Armonk, New York, United States.

Results Forty-six children were included in the study. The median age of children in our study was 6 years (range: 1 year 2 months-12 years). Undernutrition was associated with a significant increase in toxicity $(p=0.02)$. Fifty-two percent of children had evidence of toxicity, elevated transaminases being the most common. There were recurring symptoms resulting in 53 episodes of toxicities overall. Incidence of toxicity was more in the early cycles $(<3)$.

- administration

Conclusion The pre- and post-MTX hydration is an effective way to reduce toxicities with the Capizzi-style MTX and this course can be administered with ease on outpatient basis with minimal need for monitoring or admission.
\end{abstract}

DOI https://doi.org/ 10.1055/s-0041-1740442. ISSN 0971-5851.

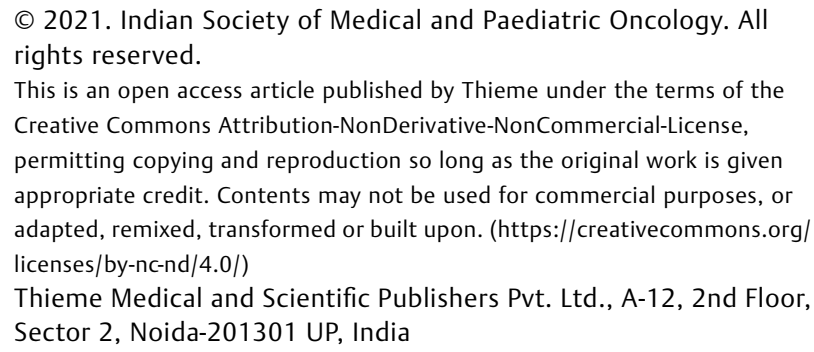

(C) 2021. Indian Society of Medical and Paediatric Oncology. All rights reserved.

This is an open access article published by Thieme under the terms of the Creative Commons Attribution-NonDerivative-NonCommercial-License, permitting copying and reproduction so long as the original work is given appropriate credit. Contents may not be used for commercial purposes, or adapted, remixed, transformed or built upon. (https://creativecommons.org/ licenses/by-nc-nd/4.0/)

Thieme Medical and Scientific Publishers Pvt. Ltd., A-12, 2nd Floor, Sector 2, Noida-201301 UP, India 


\section{Introduction}

The Capizzi-style methotrexate (MTX) has been an integral part of acute lymphoblastic leukemia (ALL) chemotherapy and is gaining importance in various subsets of leukemia. Despite the fact that it has been a part of various study protocols, the toxicity associated with escalating doses of MTX has not been studied in detail. The original Capizzi included escalating doses of MTX with L-asparaginase. From the earlier ALL studies in the United Kingdom, it was found that the dose escalation to a maximum of $300 \mathrm{mg} / \mathrm{m}$ was possible in only $10 \%$, while only a meager $40 \%$ could achieve any kind of dose escalation. ${ }^{1}$ The Indian Childhood Collaborative Leukemia (ICiCLe) protocol institutes the modified Capizzi MTX regimen for interim maintenance phase of intermediate-risk children with B-cell precursor (BCP) ALL. It includes only escalating dose of intravenous (IV) MTX with vincristine and intrathecal MTX. We have reviewed the toxicity profile encountered with the Capizzi interim maintenance in intermediate-risk ALL children from our institute treated as per the ICiCLe protocol.

\section{Materials and Methods}

The patients diagnosed with ALL with BCP type based on morphology and flow cytometry of the bone marrow and stratified to intermediate risk based on ICiCLe risk groups were included in the study. All children between 1 and 14 years' age group diagnosed with intermediate-risk ALL were included in the study. Intermediate risk as per the ICiCLe protocol constitutes children with BCP ALL with bulky disease and/or age $>10$ years and/or white blood cell count above 50,000 and/or testicular involvement with good day 8 prednisolone response ( $<1,000$ blasts in peripheral smear) and end of induction minimal residual disease (MRD) of $<0.01 \%$. Those children with T-cell ALL, poor prednisolone response and abnormal cytogenetics with BCR ABL translocation, MLL rearrangements, iAMP21, t $(17 ; 19)$, and postinduction MRD $>0.01 \%$ were treated as high risk and therefore excluded from the study. All patients treated in the year 2019 and had completed the Capizzi regimen by December 2019 were included in the study $(n=46)$. The data regarding clinical presentation, prednisolone response, biochemical, hematological parameters, MRD, and details about various toxicities encountered during the interim maintenance phase were recorded retrospectively from the patient's file in the medical record department. Hemogram and liver and renal function tests were done routinely prior to each cycle. A minimum absolute neutrophil count of 750/ $\mathrm{mm}^{3}$ and platelets $>100,000 / \mathrm{mm}^{3}$ were prerequisites for starting each Capizzi cycle. Transaminases $<2$ times upper limit of normal and a normal renal function were also looked into as a part of unit protocol prior to start each cycle. Although the ICiCLe protocol provides larger cut-off values, these modifications were made by the unit based on previous experience with such patients. Prehydration with one-pint normal saline was administered routinely, followed by IV MTX, and subsequently, one-pint normal saline was also given in an attempt to minimize toxicity. IV vincristine at 1.5 $\mathrm{mg} / \mathrm{m}^{2}$ was given on days $2,12,22,32$, and 42 along with MTX in escalating doses from 100 to $300 \mathrm{mg} / \mathrm{m}$ every 10 days (days 2, 12, 22, 32, and 42 corresponding to weeks 11-16). Two doses of intrathecal MTX (age-adjusted dosing $<2$ years: $8 \mathrm{mg}$; 2-3 years: $10 \mathrm{mg}$; and $>3$ years: $12 \mathrm{mg}$ ) were given on days 1 and 15 of the course as per the ICiCLe protocol version 1. Urine $\mathrm{pH}$ monitoring was not done routinely. Midcycle tests were not repeated unless the child was symptomatic. The clinical evidence of various toxicities as per the common terminology criteria for adverse events (NCI CTCAE v 5.0) criteria $^{2}$ such as mucositis, febrile neutropenia, vomiting, diarrhea, trend of total white blood cell counts, and neutrophils and biochemical evidence of elevated transaminases, delay in administration of chemotherapy, ability to escalate MTX in consequent courses, need for dose modification, and leucovorin rescue or antibiotics were documented. The correlation between toxicities and nutritional status was analyzed. Undernutrition was considered in those children with anthropometric values $Z$ scores $<-2$ standard deviation as per the World Health Organization (WHO) growth standards. The duration of course was considered to be 43 days, approximately the day when each child received his/her fifth cycle of MTX. This was taken so to ensure uniformity in the analysis.

\section{Statistical Analysis}

Data were entered onto SPSS worksheet and appropriate statistical methods were used to analyze the variables. Mean and median were calculated for age distribution. The incidence of toxicity was assessed with categorical variables such as gender (male/female) and undernourishment (yes/no based on the above definition). The age group was divided into two categories $(<1>3$ years) to assess its influence on the incidence of toxicity. A $p$-value of $<0.05$ was considered to be statistically significant. Chi-square test was performed to assess the significance of the categorical variables using the SPSS software version 24 for Mac, IBM Corp. Armonk, New York, United States.

\section{Results}

A total of 66 children were treated as intermediate-risk ALL based on cell of origin, cytogenetic features, prednisolone response, and MRD status during the year 2019. However, only 46 had completed their interim maintenance cycle by December 2019 and were included in the analysis. A total of 230 cycles of intermediate-dose MTX were studied for the above-mentioned toxicities. The median age of children included in the study was 6 years (range: 1 year 2 months- 12 years) with a mean of $5.7 \pm 3.5$ years. The total boys included were 26 and girls were 20 . Baseline characteristics of children treated in our unit under intermediate-risk ICiCLe protocol are shown in -Table 1. Treatment-related toxicity was noted in 53 of 230 cycles (23\%). The most common toxicities encountered in our children overall ( $n=230$ cycles) were elevated transaminases $(n=16)$, mucositis $(n=10)$, and vomiting $(n=8)$. Fever was documented in seven children, 
Table 1 Baseline characteristics of children treated as per intermediate-risk Indian Childhood Collaborative Leukemia protocols

\begin{tabular}{|l|l|}
\hline Details & Numbers \\
\hline $\begin{array}{l}\text { Total children with intermediate } \\
\text { risk all in 2019 }\end{array}$ & 66 \\
\hline Included in the study & 46 \\
\hline Median age (y) & 6 \\
\hline Gender & \\
\hline Boys & 26 \\
\hline Girls & 20 \\
\hline Average total counts (range) & 14,245 \\
\hline Flow cytometry-precursor B ALL & $(900-58,000)$ \\
\hline CD 10+ & 46 \\
\hline CD 19+ & 28 \\
\hline Aberrant myeloid markers & 43 \\
\hline Cytogenetics & 2 \\
\hline t(1; 19) & \\
\hline t(12; 21) & 2 \\
\hline Hyperdiploidy & 1 \\
\hline Others & 1 \\
\hline Normal karyotype & 3 \\
\hline $\begin{array}{l}\text { Cytogenetics failed (FISH for BCR/ABL, } \\
\text { MLL, i AMP21 negative confirmed) }\end{array}$ & 17 \\
\hline Good prednisolone response & 46 \\
\hline Current status & \\
\hline In remission and on treatment & 43 \\
\hline Relapsed & 3 \\
\hline
\end{tabular}

Abbreviations: ALL, acute lymphoblastic leukemia; B ALL, B-cell ALL; $\mathrm{BCR} / \mathrm{ABL}$, B-cell receptor/abel-like kinase translocation (Ph-positive ALL); FISH, florescent in situ hybridization; i AMP21, intrachromosomal amplification of chromosome 21; MLL, mixed lineage leukemia gene. with growth of methicillin-resistant Staphylococcus aureus in sputum culture $(n=1)$, pneumonia $(n=2)$, tooth abscess ( $n$ $=1)$, viral hepatitis $(n=1)$, and without focus $(n=2)$. Febrile neutropenia was encountered in only $2 / 230$ cycles. Six of our patients needed admission during the entire course $(13 \%)$. The incidence of various toxicities with the Capizzi regimen is shown in -Table 2. The need for IV antibiotics for infection/mucositis was prescribed in 3 of 46 (6.5\%) patients, while oral antibiotics were prescribed in 14 of 46 (30\%). We did not encounter any neurological or renal toxicity. There was no statistically significant difference in toxicity based on gender $(p=1.000)$. We found that $<3$-year-old children had a higher incidence of toxicity; however, it was not statistically significant $(p=0.06)$, while undernourishment was associated with a significantly increased incidence of toxicity ( $p=$ 0.02 ).

Although the total incidence of each of the toxicities was $23 \%$, only in $10.4 \%$ of the total cycles ( $n=24 / 230$ ), these toxicities resulted in a delay of chemotherapy. About 16 children had no delay in treatment and had completed all five cycles of the Capizzi interim maintenance in the stipulated time of approximately 43 days, while the remaining 30 children had a delay in treatment due to various side effects/default as depicted in -Fig. 1, the most common being elevated transaminases. In $52.2 \%$ of children, the delay was due to toxicity (24/46), while $13 \%$ (6/46) had a delay due to default, and the remaining $34.8 \%$ (16/46) had completed the course in stipulated time. This indicates that there were recurring toxicities in these 24 children that lead to a total of 53 episodes of toxicities overall. The average duration of the interim maintenance course in our children was 52 days (range: 43-82 days). Six patients had defaulted treatment for more than an average duration of 16 days. Since the Capizzi phase was administered on outpatient basis, an average delay of 3 days was noted even in those patients who had completed treatment by 46 days, attributed to nonworking days, difficulty in transport, and other personal reasons. In 14 children, toxicities were noted in the first three cycles,

Table 2 Incidence of various toxicities with the Capizzi interim maintenance

\begin{tabular}{|l|l|l|l|l|}
\hline Toxicity encountered & $\begin{array}{l}\text { Number of } \\
\text { episodes (53/230) }\end{array}$ & $\begin{array}{l}\text { Grade 3 or 4 toxicity/ } \\
\text { delay in CT (24/230) (\%) }\end{array}$ & $\begin{array}{l}\text { Number of } \\
\text { patients with } \\
\text { recurrent episodes } \\
(\boldsymbol{n}=\mathbf{4 6})\end{array}$ & $\begin{array}{l}\text { Number } \\
\text { of patients } \\
\text { requiring } \\
\text { admission (6/46) }\end{array}$ \\
\hline Elevated transaminases & 16 & $8(50)$ & 5 & 0 \\
\hline Mucositis & 10 & $5(50)$ & 4 & 2 \\
\hline Vomiting & 8 & 0 & 0 & 0 \\
\hline Diarrhea & 5 & 0 & 0 & 0 \\
\hline Neutropenia & 6 & $4(66.6)$ & 2 & 0 \\
\hline Thrombocytopenia & 1 & 0 & 0 & 0 \\
\hline Infection & 7 & $7(100)$ & 0 & 4 \\
\hline CNS toxicity & 0 & 0 & 0 & 0 \\
\hline Renal toxicity & 0 & 0 & 0 & 0 \\
\hline
\end{tabular}

Abbreviations: CNS, central nervous system; CT, computed tomography. 


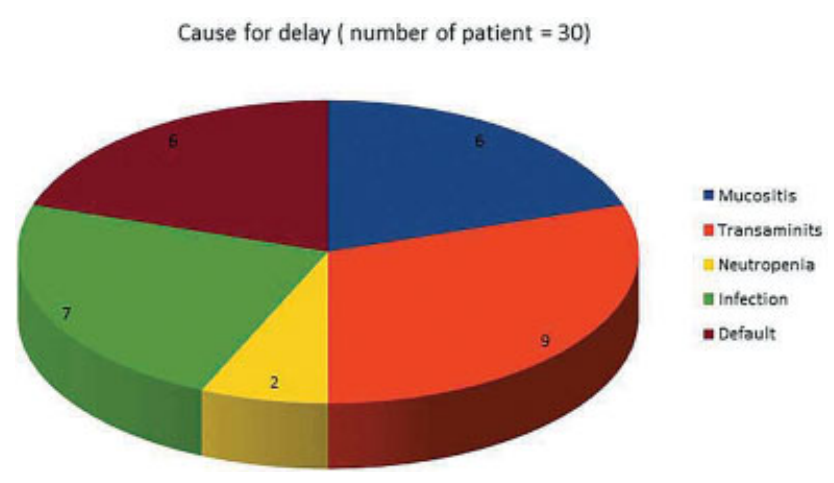

Fig. 1 Cause for delay (number of patient $=30$ ).

while in 10 children, the toxicities were seen in fourth/fifth cycle.

The dose was capped at $200 \mathrm{mg} / \mathrm{m}^{2}$ for three children who had severe mucositis and persistent elevation of transaminases. The rest (93\%) of the children received escalating doses of IV MTX with appropriate dose escalation. Ursodeoxycholic acid was used for those children with elevated transaminases.

\section{Discussion}

The practice of escalating dose of MTX began with the Children's cancer group (CCG) 1991 study where standardrisk patients were randomized to receive oral versus escalating doses of MTX during the interim maintenance phase. ${ }^{1}$ Patients with central nervous system $\left(\mathrm{CNS}^{2} ;<5\right.$ lymphocytes in cerebrospinal fluid with cytospin analysis positive for leukemic blasts), involvement also seemed to have benefited from this randomization as their outcomes improved to 85 from $63 \%$ in previous studies. Dexamethasone was the only steroid used in the protocol which provides good CNS penetration and could have added on to the improved survival. ${ }^{3}$ In the recent AALL0434 trial, a randomization between the Capizzi-style MTX and high-dose MTX (HD MTX) for T-cell ALL (T ALL) was performed where the Capizzi regimen had a better disease-free survival of 91.5 versus $85.3 \%$. However, cranial irradiation was included in both the groups at 2 and 7 months, respectively. ${ }^{4}$ These surprisingly better results in the Capizzi arm that was explained with differential sensitivity of the blasts to use of MTX without leucovorin rescue, the lack of use of L-asparaginase and also the timing of irradiation which could have been the reasons behind improved outcomes with less intense MTX dosing. The other studies which have shown comparable results of the Capizzi and HD MTX in T ALL have utilized an intensified intrathecal therapy up to 28 doses in males and 21 doses in females. ${ }^{5}$ Thus, the Capizzi regimen is increasingly gaining importance in various aspects of ALL management. Reports of comparable toxicity and ease of administration of HD MTX regimens exist, while the literature on toxicity encountered with the Capizzi MTX has been addressed by very few studies in the past.

Many times, the outpatient-based administration of drug with a lack of structured monitoring in between cycles results in delayed presentation and increased incidence of treatment-related toxicity. ${ }^{6}$ The traditional Capizzi interim maintenance used by the United Kingdom and Children's Oncology Group studies included L-asparaginase which has been omitted in the ICiCLe protocol, based on the report from AALL0232 study which showed increased toxicity than benefit from this combination. On the contrary, there are also some reports that suggest L-asparaginase helps in attenuating the MTX-related toxicity. ${ }^{7}$

Gender-related changes in the incidence of MTX toxicity were observed in early osteosarcoma studies which were attributed to the prechemotherapy erythrocyte folate levels. Some pediatric ALL studies have also demonstrated an increased incidence of toxicity and treatment-related mortality in girls. ${ }^{8}$ There was no difference in the incidence of toxicity based on gender in our study $(p=1.000)$. Several polymorphisms can affect MTX dosing and related toxicity. The MTHFR C677T polymorphism has been associated with an increased risk of MTX-induced severe hepatic and gastrointestinal toxicities in Caucasian adults being treated for cancer. ${ }^{9}$ The expression of multidrug resistance gene polymorphisms, like ABCC2, affects folate metabolism and causes MTX toxicity. The ALT levels have remained a surrogate marker to assess toxicity in the study by Jastaniah et al. ${ }^{5}$ The highest value of ALT documented in our study was 812 $\mathrm{U} / \mathrm{L}$, none of the children developed deranged prothrombin time (PT)/activated partial thromboplastin time (APTT) or signs of liver failure. Ursodeoxycholic acid was routinely used in children with elevated transaminases and response to therapy in the form of decreasing trend of liver enzymes was documented in $100 \%$ of the children.

A tertiary care center from North India reported comparable toxicities with the HD MTX and Capizzi MTX. Myelosuppression was the most common encountered, while skin and nephrotoxicity were seen only in the high-dose arm. ${ }^{10}$ Twelve of our 46 children were undernourished and increased incidence of toxicity was noted in this subgroup. Undernourished children had increased incidence of toxicity in their study as well, with incidence of 44 versus $30 \%$ in wellnourished children. The dysfunctional liver status in malnutrition probably contributed to increased toxicity in these children. In the AALL0232 study, patients receiving HD MTX had a lower incidence $(5.2 \%)$ of febrile neutropenia than those receiving the standard dose regimen (8.2\%). There were no differences in other significant toxicities in the study. ${ }^{11}$ The modification of the original Capizzi and avoidance of L-asparaginase are expected to lessen the toxicity associated with this regimen in the ICiCLe protocol. We had only 2 of 230 episodes of febrile neutropenia $(0.8 \%)$, while the other 5 fever episodes were non neutropenic. We attribute the lower incidence of toxicity to the method of administration of MTX. Pre- and post-MTX hydration has been an easy and effective way administering MTX without requiring admission or urine $\mathrm{pH}$ monitoring. Our treatment-related toxicity was slightly lower at $23 \%$ compared with $28 \%$ in the above-mentioned study. ${ }^{10}$ While febrile neutropenia and thrombocytopenia were the most common toxicities encountered in their children approximately $20 \%$ each, our 
profile mainly constituted hepatotoxicity and mucositis. Both of these were documented in $<10 \%$ of their cycles. There are isolated reports of severe nephrotoxicity with intermediate-dose MTX at $200 \mathrm{mg} / \mathrm{m}$ requiring folinic acid rescue and dialysis. ${ }^{12}$ However, none of our children had renal function derangement. Some studies have even suggested that MTX dose must be tailored based on the genetic polymorphisms. ${ }^{13}$ A strong association between preexisting folate deficiency, longer time for bone marrow recovery, hypoalbuminemia, and MTX toxicity has been well established by Tandon et al. ${ }^{14}$

\section{Conclusion}

We thereby would like to highlight that the Capizzi regimen can be administered on outpatient basis with ease and tolerable toxicity. Pre- and post-MTX hydration for these children decreases the incidence of renal toxicity without the need for admission and subsequent monitoring. Dose escalation must not be withheld for mild toxicities as improved tolerance to MTX in the latter cycles was observed in many of our children. Good hydration, patient education for oral hygiene, and early appropriate management with oral or IV agents can help in curbing several toxicities at an early stage and thereby aid the administration of appropriate dosage on time.

\section{Ethics}

Kidwai Memorial Institute of Oncology Ethics Committee provided a waiver in view of retrospective nature of the study and thereby permitted the analysis and publication of the patient data without obtaining a formal approval on May 31, 2021. The procedures followed were in accordance with the ethical standards of the responsible committee on human experimentation and with the Declaration of Helsinki 1964, revised in 2013.

\section{Financial Support and Sponsorship}

None.

\section{Conflicts of Interest}

There are no conflicts of interest.

\section{References}

1 Hough R, Rowntree C, Goulden N, et al. Efficacy and toxicity of a paediatric protocol in teenagers and young adults with Philadelphia chromosome negative acute lymphoblastic leukaemia: results from UKALL 2003. Br J Haematol 2016;172(03):439-451
2 Common Terminology Criteria for Adverse Events (CTCAE). Protocol Development cancer Therapy Evaluation Program (CTEP). Available at: https://ctep.cancer.gov/protocoldevelopment/electronic_applications/docs/CTCAE_v5_Quick_Reference_5x7.pdf. Accessed July 12, 2021

3 Matloub Y, Bostrom BC, Hunger SP, et al. Escalating intravenous methotrexate improves event-free survival in children with standard-risk acute lymphoblastic leukemia: a report from the Children's Oncology Group. Blood 2011;118(02):243-251

4 Capizzi-style methotrexate improves survival in pediatric T-cell acute lymphoblastic leukemia. Available at: https://www.healio. com/news/hematology-oncology/20180910/capizzistyle-methotrexate-improves-survival-in-pediatric-tcell-acute-lymphoblastic-leukemia. Accessed on June 17, 2020

5 Jastaniah W, Elimam N, Abdalla K, AlAzmi AA, Aseeri M, Felimban S. High-dose methotrexate vs. Capizzi methotrexate for the treatment of childhood T-cell acute lymphoblastic leukemia. Leuk Res Rep 2018;10; 44-51

6 Methotrexate vs. Capizzi methotrexate examined in youth with BALL. Available at: https://www.empr.com/home/news/drugnews/methotrexate-vs-capizzi-methotrexate-examined-inyouth-with-b-all/. Accessed on June 17, 2020

7 Lobel JS, O’Brien RT, McIntosh S, Aspnes GT, Capizzi RL. Methotrexate and asparaginase combination chemotherapy in refractory acute lymphoblastic leukemia of childhood. Cancer 1979;43 (03):1089-1094

8 Moulik NR, Kumar A, Agrawal S, Mahdi AA, Kumar A. Effect of folate status and methylenetetrahydrofolate reductase genotypes on the complications and outcome of high dose methotrexate chemotherapy in north Indian children with acute lymphoblastic leukemia. Indian J Med Paediatr Oncol 2016;37(02):85-89

9 Zhao M, Liang L, Ji L, et al. MTHFR gene polymorphisms and methotrexate toxicity in adult patients with hematological malignancies: a meta-analysis. Pharmacogenomics 2016;17(09): 1005-1017

10 Mandal P, Samaddar S, Chandra J, Parakh N, Goel M. Adverse effects with intravenous methotrexate in children with acute lymphoblastic leukemia/lymphoma: a retrospective study. Indian J Hematol Blood Transfus 2020;36(03):498-504

11 High-dose methotrexate improves survival in high-risk B-cell precursor acute lymphoblastic leukemia. Available at: http:// www.ahdbonline.com/issues/2011/august-2011-vol-4-no-4special-issue/782-article-782. Accessed on July 19, 2020

12 Stark AN, Jackson G, Carey PJ, Arfeen S, Proctor SJ. Severe renal toxicity due to intermediate-dose methotrexate. Cancer Chemother Pharmacol 1989;24(04):243-245

13 Liu SG, Gao C, Zhang RD, et al. Polymorphisms in methotrexate transporters and their relationship to plasma methotrexate levels, toxicity of high-dose methotrexate, and outcome of pediatric acute lymphoblastic leukemia. Oncotarget 2017;8(23): 37761-37772

14 Tandon S, Moulik NR, Kumar A, Mahdi AA, Kumar A. Effect of pretreatment nutritional status, folate and Vitamin B12 levels on induction chemotherapy in children with acute lymphoblastic leukemia. Indian Pediatr 2015;52(05):385-389 